\title{
Food and Drug Interaction: Consequences for the Nutrition/Health Status
}

\author{
Dieter Genser \\ Gesundheit Österreich GmbH (GÖG)/Österreichisches Bundesinstitut für Gesundheitswesen (ÖBIG) - \\ Vergiftungsinformationszentrale, Wien, Austria
}

\section{Key Words}

Food-drug interactions • Drug-nutrient interactions •

Nutritional status

\begin{abstract}
Food-drug interactions are defined as alterations of pharmacokinetics or pharmacodynamics of a drug or nutritional element or a compromise in nutritional status as a result of the addition of a drug. Elderly patients are particularly at risk because more than $30 \%$ of all the prescription drugs are taken by this population. Failure to identify and properly manage drug-nutrient interactions can lead to serious consequences. For instance, drug-nutrient interactions can result in reduced absorption of certain oral antibiotics and may lead to suboptimal antibiotic concentrations at the site of infection. This predisposes the patient to treatment failure. Induction or inhibition of enzymes in the gut by nutrients may lead to a significant change in oral bioavailability of drugs or vice versa. For example, grapefruit juice is a selective intestinal CYP3A4 inhibitor. The overall exposure of some drugs can be increased by more than fivefold when taken with grapefruit juice and increase the risk of adverse effects. The use of certain drugs may affect Gl tract function and may lead to a loss of bodily electrolytes and fluid. Limiting drug prescriptions to essential medications for as short a period as possible and periodic re-evaluations of the treatment chosen are essential to minimize adverse drug-nutrient interactions.

Copyright $\odot 2008$ S. Karger AG, Basel
\end{abstract}

\section{Introduction}

In the past, food-drug interactions (drug-nutrient interactions) were limited to determine whether meal intake would impair drug absorption and therefore influence the bioavailability of a drug. Later, it was shown that enteral feeding could also affect the pharmacokinetics of drugs. Until recently, these interactions were not clearly defined and characterized in the literature. Properly designed studies on the epidemiology of food-drug interactions and standardized management approaches and consensus toward specific drug-nutrient interactions are missing [1].

Drug-nutrient interactions are now defined as alterations of pharmacokinetics or pharmacodynamics of a drug or nutritional element or a compromise in nutritional status as a result of the addition of a drug. Pharmacokinetics refers to the quantitative description of drug disposition, which includes absorption, distribution, metabolism, and excretion. Pharmacodynamics refers to the physiologic or clinical effects of a drug [2].

Food-drug interactions can result in two main clinical effects: either a decreased bioavailability of a drug, which predisposes to treatment failure, or an increased bioavailability, which increases the risk of adverse events and may even precipitate toxicities.

Patient populations who have increased risks of suffering from adverse events associated with drug-nutrient interactions are elderly patients, patients with cancer and/

\section{KARGER}

Fax +4161306 1234

E-Mail karger@karger.ch

www.karger.com
(C) 2008 S. Karger AG, Basel

0250-6807/08/0525-0029\$24.50/0

Accessible online at:

www.karger.com/anm
DDr. Dieter Genser

Gesundheit Österreich GmbH (GÖG)/Österreichisches Bundesinstitut für Gesundheitswesen (ÖBIG) - Vergiftungsinformationszentrale, Leitstelle 6Q Währinger Gürtel 18-20, AT-1090 Wien (Austria), Tel. +43 140400/2222

Fax +43 $140400 / 4225$, E-Mail Dieter.Genser@meduniwien.ac.at 
or malnutrition, gastrointestinal tract dysfunctions, acquired immunodeficiency syndrome, those receiving enteral nutrition and transplant recipients. Elderly patients are particularly at risk because more than $30 \%$ of all the prescription drugs are taken by this population [1]. Among the most common health problems in elderly people are spine or back diseases, arthritis/rheumatism, heart diseases, hypertension and depression. Accordingly, the most common drugs taken by this age group are non-steroidal anti-inflammatory drugs, antihypertensives, and antidepressants [3]. Multiple underlying chronic diseases require long-term nutritional and pharmacotherapeutic interventions. The practice of polypharmacy (i.e. using multiple drugs to manage different disease states) increases patients' risk of drug-nutrient interactions [1].

In addition, elderly patients are more likely to experience adverse events because several physiologic functions are reduced with age, such as the sense of taste and smell, chewing and swallowing, and gut motility. Pharmacokinetic peculiarities associated with aging are absorption (changes in gastric $\mathrm{pH}$, decreased gastrointestinal and splanchnic blood flow), distribution (decreased lean body mass, water, serum albumin concentration and binding serum proteins; increased total body fat, increased bloodbrain barrier permeability) and elimination (reduced renal function). Therefore, drug bioavailability, volume of distribution, clearance and half-life of drugs are modified in the elderly. Water-soluble drugs become more concentrated and fat-soluble drugs may have longer halflives because of a slower release of the drug from fatty tissues. The activity of the cytochrome $\mathrm{P} 450$ oxidase systems are also modified in older patients. As many of these age-related factors are difficult to predict, the ideal prescription of a drug is complex [3].

\section{Types of Drug-Nutrient Interactions}

Based on the nature and the mechanisms, four types of drug-nutrient interactions are categorized. Type I are ex vivo bioinactivations, which refer to interactions between the drug and the nutritional element or formulation through biochemical or physical reactions, such as hydrolysis, oxidation, neutralization, precipitation or complexation. They usually occur in the delivery device. Type II interactions affect absorption. They cause either an increase or decrease of the oral bioavailability. The precipitant agents may modify the function of enzymes (type A interactions) or transport mechanisms (type B interactions) that are responsible for biotransformation. Complexation, binding, and/or other deactivating processes occur in the gastrointestinal tract (type $\mathrm{C}$ interaction) and reduce absorption. Type III interactions affect the systemic or physiologic disposition and occur after the drug or the nutritional element has been absorbed from the gastrointestinal tract and entered the systemic circulation. Changes of the cellular or tissue distribution, systemic transport, or penetration to specific organs or tissues can occur. Type IV interactions refer to the elimination or clearance of drugs or nutrients, which may involve the antagonism, impairment or modulation of renal and/or enterohepatic elimination [1].

\section{Drug-Nutrient Interactions: Examples and Practical Relevance}

Type I interactions are most common with drugs and nutrients administered intravenously. There are several recommendations to minimize type I drug-nutrient interactions. For instance, drugs should not be mixed directly with feeding enteral or parenteral formulas. Tubes should be flushed with water before and after drug administration. Preformulated oral solutions or suspensions should be preferred instead of crushing tablets when administering drugs through enteral feeding tubes. For drugs with a narrow therapeutic range, monitoring drug levels should be taken into consideration.

\section{Meal Intake and Drug Absorption}

An example of type II interactions is the influence of oral absorption of a drug by concurrent meal intake. The rate of absorption, the magnitude of the absorption, or both can be changed. Meal intake stimulates gastric and intestinal secretions, which usually improve the dissolution of drugs and facilitate absorption. Meals with higher fat content stimulate the release of bile salts, which increase the intestinal uptake of highly lipophilic drugs or of substances which require bile salts for optimal absorption. In addition, a high fat content of the food also stimulates the release of cholecystokinin, which slows gastrointestinal motility and increases the contact time between the drug and the intestine and possibly also absorption. However, the potential physicochemical interactions mentioned above, the potential binding of drug and food contents, the dose of the drug administered, and the composition of the meals make drug absorption in the pres- 
ence of food unpredictable in specific cases. For that reason the bioavailability of drugs should be tested with and without concurrent meal intake. It has been shown that certain drugs should be taken with food to maximize absorption. Among these drugs are the antibiotics cefuroxime and erythromycin ethylsuccinate, the HMGCoA-reductase inhibitor lovastatin, and lithium (used for psychiatric diseases). On the other hand, several drugs should not be taken with food to allow optimal absorption: the antibiotics ampicillin, ciprofloxacin, doxycycline or tetracycline; captopril (used for elevated blood pressure) or the HIV-protease inhibitor indinavir [1].

It should be mentioned, however, that delayed absorption of a drug by food does not necessarily lead to reduced absorption and that pharmacokinetic changes do not necessarily have clinically relevant effects [4].

\section{Food Intake and Drug Metabolism: Examples}

The metabolism of orally ingested drugs before reaching the systemic circulation ('first-pass metabolism' or nowadays 'presystemic clearance') has been shown to have clinically relevant influences on the potency and efficacy of drugs. Both the intestine and the liver account for the presystemic metabolism in humans. One of the major groups of drug-metabolizing enzymes is the cytochrome P450 (CYP) 3A4 enzymes. Grapefruit juice is a classic example of a selective intestinal CYP 3A4 inhibitor. It causes a type IIA interaction with certain drugs by deactivating and destroying intestinal CYP3A4 enzymes. The overall exposure of some drugs can be increased by more than fivefold when taken with grapefruit juice. It has been shown that furanocoumarin derivatives in the juice are primarily responsible for enzymatic inhibition [5]. The onset of the interaction is immediate and the magnitude of the enzymatic inhibition increases with repeated grapefruit juice consumption. The effect continues for several days after the discontinuation of grapefruit juice consumption. The increased oral absorption of certain drugs can lead to increased risk for adverse reactions of the specific drugs. For instance, drowsiness and prolonged sedation may result from taking the tranquillizers diazepam or midazolam in combination with grapefruit juice. Hypotension can result in people treated with calcium-channel blockers (nifedipine, felodipine, verapamil). Patients taking the antibiotic erythromycin and consuming grapefruit juice have an increased risk for cardiovascular symptoms, including cardiac dysrhythmias. Other drugs with increased absorption when taken with grapefruit juice are atorvastatin, cyclosporine and sertralin [1].

Drugs may also be associated with altered metabolic function or macronutrient status. Some atypical antipsychotics (clozapine, olanzapine, risperidone) are associated with glucose intolerance. Particularly when risk factors such as an underlying diabetic condition or an increase in weight are present or certain other medications are concomitantly used, more frequent monitoring of blood glucose should be considered. Alternatively, in these situations antipsychotics for which no association with glucose intolerance has been demonstrated (haloperidol, chlorpromazine) should be preferred when possible [6].

\section{Drugs Interfere with Food Intake}

Medications can lead to altered food choices. Many drugs are reported to directly affect the sense of taste and smell and some drugs themselves have an unpleasant taste that might interfere with food intake [7].

It is well known that chemotherapeutic drugs (and to a lesser extent also other drugs) induce nausea and vomiting and therefore are associated with an increased risk for malnutrition. However, also drugs which decrease acute and delayed emesis (e.g. aprepitant) may have adverse effects such as fatigue, dysphagia, taste disturbance, constipation, diarrhea or anorexia and could therefore by itself adversely affect the nutritional status [8].

Limiting drug prescriptions to essential medications for a short period as possible and periodic re-evaluations of the treatment chosen are essential to minimize adverse drug-nutrient interactions [4].

\section{Conclusion}

In general, most data on the pharmacodynamics and pharmacokinetics of drugs in special patient populations pertain to renal impairment and hepatic dysfunction. Less data address drug pharmacodynamics and pharmacokinetics in the elderly, and these drug characteristics are only infrequently assessed in regard to malnourished patients. The paucity of information on the influence of the nutritional status (e.g. malnutrition, obesity, and micronutrient deficiencies) on drugs has been criticized [8]. On the contrary, primarily data regarding the influence of drug-nutrient interactions on the nutritional status are also missing. 
The selection of appropriate pharmacotherapy for elderly people has been recognized as a challenging and complex process and has become an important public health issue [9]. Moreover, the increased risk of elderly patients for interactions of drug-food and drug-nutritional status, but also for drug-alcohol and drug-herbal products is being discussed. Approaches to categorize drug interactions, as well as strategies for detection, management and prevention have been proposed. Computerassisted drug interaction software can serve as a reference source. However, a multiprofessional team-based approach, including physicians, pharmacists, and nurses has been proposed [10]. Nutritional scientists could also add valuable knowledge.

\section{Disclosure Statement}

None declared.

\section{References}

1 Chan LN: Drug-Nutrient Interactions; in Shils ME, Shike M, Ross AC, Caballero B, Cousins RJ (eds): Modern Nutrition in Health and Disease. Baltimore, Lippincott Williams \& Wilkins, 2006, pp 1540-1553

$\checkmark 2$ Chan LN: Drug-nutrient interaction in clinical nutrition. Curr Opin Clin Nutr Metab Care 2002;5:327-332.

$\checkmark 3$ Akamine D, Filho MK, Peres CM: Drug-nutrient interactions in elderly people. Curr Opin Clin Nutr Metab Care 2007;10:204310.

4 Schmidt LE, Dalhoff K: Food-drug interactions. Drugs 2002;62:1481-1502.
Paine MF, Widmer WW, Hart HL, Pusek SN, Beavers KL, Criss AB, Brown SS, Thomas BF, Watkins PB: A furanocoumarin-free grapefruit juice establishes furanocoumarins as the mediators of the grapefruit juice-felodipine interaction. Am J Clin Nutr 2006;83: 1097-1105.

-6 Hedenmalm K, Hägg S, Stahl M, Mortimer Ö, Spigset O: Glucose intolerance with atypical antipsychotics. Drug Safety 2002;25: 1107-1116.

7 Brownie S: Why are elderly individuals at risk of nutritional deficiency? Int J Nurs Pract 2006;12:110-118.
$>8$ Santos CA, Boullata JI: An approach to evaluating drug-nutrient interactions. Pharmacotherapy 2005;25:1789-1800.

$\checkmark 9$ Spinewine A, Schmader KE, Barber N, Hughes C, Lapane KL, Swine C, Hanlon JT: Appropriate prescribing in elderly people: how well can it be measured and optimised? Lancet 2007;370:173-184.

10 Mallet L, Spinewine A, Huang A: The challenge of managing drug interactions in elderly people. Lancet 2007;370:185-191. 